\title{
Las operaciones cognitivas empleadas en la producción de textos académicos
}

\section{Cognitive operations used in the production of academic texts}

Recibido: 17 de octubre de 2019 | Aprobado: 11 de diciembre de 2019

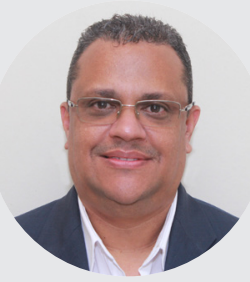

JOSÉ ALEJANDRO RODRÍGUEZ NÚÑEZ* PONTIFICIA UNIVERSIDAD CATÓLICA

MADRE Y MAESTRA, REPÚBLICA DOMINICANA joserodriguezn@pucmm.edu.do

\section{Resumen}

Conscientes de las implicaciones que conlleva el proceso de escritura y el reto que asumen nuestros estudiantes para lograrlo, proponemos el siguiente artículo con la finalidad de exponer el rol que desempeñan las operaciones mentales (cognitivas) en relación con la producción escrita, considerada como un acto comunicativo complejo en virtud de los procedimientos lingüísticos, pragmáticos y cognitivos que intervienen en dicho acto, que a la vez se convierte en una práctica social. Utilizamos la deducción como método estratégico, de manera que, a partir de premisas generales, a la luz de referentes teóricos y metodológicos, experiencias y acciones concretas, ofrecemos una propuesta basada en principios didácticos y lingüísticos que favorezca y garantice procesos cognitivos para la redacción destinada a satisfacer necesidades e intenciones comunicativas inherentes a contextos académicos. Se espera que su aplicación impacte las estrategias y actividades de aprendizaje de la escritura, permitiendo que el docente provoque espacios críticos, analíticos y reflexivos en el aula, de modo que la asignatura sea un espacio de acción y construcción de conocimientos por medio de la producción escrita.

Palabras clave: procesos; producción escrita; procesos cognitivos; didáctica de la escritura.

\section{Abstract}

Being aware of the implications of the writing process and the challenge assumed by our students to achieve it, we propose the following article aiming to expose the role played by mental (cognitive) operations in relation to the written production, considered as a complex communicative act by virtue of the linguistic, pragmatic and cognitive processes involved in this act, which at the same time becomes a social practice. Being this a theoretical-methodological proposal, we use deduction as a strategic method, so that, from general premises, and in light of theoretical and methodological references, experiences and concrete actions, we offer a proposal based on didactic and linguistic principles that favor and guarantee cognitive writing processes destined to satisfy communication needs and intentions inherent to academic contexts. Its application is expected to impact the learning strategies and writing activities, allowing the teacher to

\footnotetext{
Postgrado y Maestría en Lingüística Aplicada por el Instituto Tecnológico de Santo Domingo (INTEC). Doctor en Estudios del Español: Lingüística y Literatura por la Pontificia Universidad Católica Madre y Maestra (PUCMM). Profesor instructor y director de la Escuela de Lenguas. Para contactar al autor: JoseRodriguezN@pucmm.edu.do
}

ISSN (en línea): 1814-4152 / Sitio web: http://cuaderno.pucmm.edu.do CÓMO CITAR: Rodríguez, J. (2020). Las operaciones cognitivas empleadas en la producción de textos académicos. Cuaderno de Pedagogía Universitaria, Vol. 17, n. ${ }^{\circ} 33$, enero-junio, pp. 94-103 
provoke critical, analytical and reflective spaces in the classroom, so that the class is a space for action and knowledge building through written production.

Keywords: processes, written production, cognitive processes, didactics of writing.

\section{Introducción}

Los estudios publicados en la última década sobre la producción escrita en la universidad manifiestan un fuerte énfasis en el proceso y no en el producto. Natale (2012) y Cisneros (2014) sostienen que la producción de un texto no es espontánea ni mecánica, ya que no es el resultado de la transcripción del lenguaje oral. En efecto, la escritura, especialmente la académica, exige dedicación y esfuerzo para organizar las ideas, la planificación y la revisión de sucesivas versiones. Es por esto que el acto de escribir promueve el desarrollo del pensamiento y la reflexión sobre los propios saberes. Al respecto, Carlino (2006) ha señalado que la escritura posee un valor epistémico, es decir, que genera nuevos conocimientos, resultado del establecimiento de nuevas relaciones cognitivas. Es por esto que en la formación universitaria la tarea de escribir adquiere un papel central, ya que resulta imprescindible no solo para la transmisión de los saberes construidos en el marco de las distintas disciplinas, sino también para la generación de nuevos conocimientos (Carlino y Martínez, 2009). A esto le sumamos la promoción del estudiantado, conforme al adecuado dominio reflejado en la producción textual. Es así como en la dinámica procesual de enseñanza y aprendizaje, orientada al desarrollo de la capacidad comunicativa de producción escrita, el énfasis recae en la aplicación de estrategias de resolución de problemas de producción, basadas en la capacitación de los estudiantes con miras a la obtención de un control adecuado sobre los procesos de producción textual. Esto así, en virtud de la complejidad e implicaciones mentales que subyacen a los procesos de escritura. (Camps, 2003).

Generalmente la resolución de problemas en que se enfrascan los docentes y estudiantes giran en torno al poco dominio de la normativa lingüística, sin embargo, Casanny (1999) sostiene que no basta con que los escritores conozcan el código escrito, pues para escribir bien, los autores deben saber utilizarlo en una situación concreta; tienen que haber desarrollado procesos de composición de textos, situación que se traduce a una adecuada contextualización basada en procesos cognitivos (conocimientos) y metacognitivos (acciones estratégicas), además de recursos pragmáticos, lingüísticos, sociolingüísticos, psicolingüísticos y textuales, en que caben preguntas sobre qué escribir, para quién, para qué, con qué objetivo y cómo hacerlo. En definitiva, no basta con un conocimiento o saber mecanicista o instrumental de la escritura, mucho menos con saberes prescriptivos que, a pesar de ser necesarios, no son exclusivos ni decisivos para un acto de comunicación eficaz.

De las investigaciones sobre la enseñanza y el aprendizaje de la expresión escrita, una de las líneas se enfoca de manera particular en los procesos cognitivos o de pensamiento implicados en la escritura y en la necesidad de planificar convenientemente las tareas relacionadas con la producción de textos. Los trabajos de Flower y Hayes (1980), investigadores sobre las teorías de la escritura, han identificado los procesos psicológicos y las correspondientes actividades llevadas a cabo durante el desarrollo de la tarea de escribir, lo que implica una serie de operaciones mentales que incluyen procedimientos cognitivos, que a la vez están condicionadas por factores afectivos, sociales y físicos. Dichos procesos, desde nuestro punto de vista, implican e incluyen una gestión docente sistemática, con miras al alcance de unas competencias específicas de producciones escritas, cuyas etapas han de ser planificadas y ejecutadas tras un conocimiento lingüístico y pragmático al servicio de resultados comunicativos confiables, oportunos y pertinentes.

Es así como a partir de esta indagación se espera mostrar el beneficio que ofrece el desarrollo de destrezas cognitivas para la producción escrita, conforme a los géneros discursivos vinculadas a las distintas disciplinas y contextos académicos de la 
educación superior. Para esto, nos hemos basado en teorías, métodos y estrategias que contribuyen al desarrollo de los procesos de las producciones escritas dirigidos a satisfacer necesidades e intenciones comunicativas inherentes a los distintos contextos y ámbitos académicos. Así que, iniciaremos el desarrollo de este artículo destacando el rol social que tiene la escritura, como sus distintas funciones, lo que nos llevará a asumir uno o varios conceptos de lo que implica y significa escribir. En una segunda parte del estudio presentaremos los niveles de las habilidades cognitivas y los distintos modelos o concepciones para la didáctica de la escritura. Finalmente, ofrecemos una propuesta a partir de procesos metodológicos para desarrollar competencias cognitivas implicadas en la producción escrita.

\section{Rol de la escritura como práctica social}

En su manual sobre los sistemas de escritura en el mundo, Cisneros (2014) inicia con la siguiente interrogante: ¿Qué es escribir? Quizás para responder a esta interrogante habría que establecer distinciones entre la oralidad y la escritura, tomando en cuenta que la primera es universal, presente en todas las civilizaciones. En cambio, la escritura es un hecho claramente cultural, instrumento creado por la humanidad para mejorar su organización social: comunicarse a distancia, establecer formas de control grupal o acumular los saberes e inaugurar la historia en el sentido actual.

Desde el punto de vista social, Avendaño y Desinano (2007) nos refieren el valor y prestigio otorgado al discurso escrito, considerado como vehículo de la expresión política, jurídica y administrativa (instancias reguladoras de la vida social); de la expresión cultural (literatura, ciencia y arte) y de la comunicación periodística. Al respecto, Coulmas (2006) sostiene que, en una sociedad alfabetizada, la lengua escrita adquiere vida propia, desarrolla orientaciones parcialmente independientes y se usa para diferentes propósitos, llegándose, incluso, a considerársele superior a la modalidad oral en distintos aspectos, a pesar de que los lingüistas entienden que la modalidad escrita no es superior a la oral, sino diferente $\mathrm{y}$, hasta cierto punto, secundaria. Sin embargo, Calsamiglia y Tusón
(2009) consideran que el conjunto de funciones de conservación, oficialidad, difusión pública y medio de expresión de ciencia y cultura han otorgado al texto escrito un prestigio social único, pues sus funciones cognitivas han potenciado el desarrollo intelectual, la reflexión y elaboración mental a partir de las funciones metalingüísticas, referencial y poética del lenguaje.

En consecuencia, la escritura en sociedad requiere la apropiación del dominio de un conjunto de estrategias y procesos complejos y reflexivos entre los que cabe destacar la selección y ordenación de la información, sumados a estrategias cognitivas de generación de ideas, de revisión y reformulación. Esto no implica descuidar, bajo ningún concepto, tipologías y géneros discursivos diversos, pues como señala Graves (2002), escribir es, ante todo, una actividad social, de ahí que lo que escribimos, cómo escribimos y a quién escribimos está modelado por convenciones sociales y por nuestra historia de interacción social.

Los géneros discursivos, vinculados a las distintas disciplinas o áreas del conocimiento, son fundamentales para una didáctica de la escritura. En ellos se reconoce y admite la transversalidad de la escritura. Escribimos en todas las áreas, con distintas intenciones, desde diversas situaciones y necesidades comunicativas. En este sentido, desarrollar procesos de producción escrita implica una disposición disciplinar al servicio de un abanico de géneros, subgéneros y tipologías textuales diversas. Por esto, es preciso destacar que la pragmática promueve el uso comunicativo de la lengua en un contexto determinado en que se producen actos comunicativos concretizados en textos o discursos. Además, Álvarez (2005) asume la valoración del texto como unidad de comunicación acompañada de una tipología textual que abarca el estudio de la diversidad textual y géneros puestos de manifiesto en los intercambios comunicativos como producto de la interacción social.

Boeglin (2008) corrobora que aprender a escribir implica el dominio de los géneros discursivos para así obtener los objetivos deseados. También implica la posesión de conocimientos culturales sobre los principales usos lingüísticos de una esfera social, de modo que se pueda manejar el significado que adquieren las palabras al usarse 
en contextos nuevos. Es por esto que es de considerarse la variación de la escritura, según el dialecto y registro. De modo que aprender a escribir, conforme a los distintos escenarios discursivos en las academias, significa aprender a elegir la variedad y el registro adecuado en cada contexto, en cada disciplina, si somos conscientes de que todo escrito posee unos rasgos característicos relacionados directamente con las funciones del acto de escribir en la cotidianidad. En efecto, Carlino (2006) destaca el énfasis de la escritura a través de las disciplinas en educación superior y considera la lengua escrita como una herramienta epistémica. Para esto, sugiere el desarrollo de unos procesos cognitivos al alcance de los géneros académicos más demandados, tales como resúmenes, reseñas, ensayos, artículos, comentarios de textos, entre otros.

Con relación a la taxonomía de funciones lingüísticas, Camps (2003) distingue dos categorías funcionales del escrito en el nivel epistémico. Una primera categoría contempla el uso heurístico, que se refiere, específicamente, a la construcción de un nuevo conocimiento. Y una segunda categoría hace referencia al aspecto imaginativo, relativo a los usos más creativos y/o artísticos de la escritura. Además, se incluye otra función denominada control social que contiene todos y cada uno de los textos de carácter organizativo, vinculados a las comunidades lingüísticas. Otros tipos de funciones son clasificadas en intrapersonales e interpersonales. En lo que concierne a las funciones intrapersonales, el autor del escrito y su destinatario son la misma persona. En esta función la escritura representa una herramienta de trabajo destinada a desarrollar actividades personales, académicas o profesionales. (Cisneros, 2014).

En lo que respecta a la clasificación de funciones interpersonales, a diferencia de las funciones intrapersonales, el autor escribe para otros, ya sea para un grupo, una asociación o una comunidad específica. Así, la escritura pasa a ser un instrumento de actuación social destinada a influir, informar, ordenar, etc. Esta macro-función de la escritura va acompañada de otras funciones, como la comunicativa, la organizativa y la lúdica o estética. En lo que respecta a la función comunicativa, interactuamos en circunstancias nuevas y diversas, tanto en tiempo como en espacio, sumado a esto la precisión, la cortesía y la adecuación o pertinencia del escrito. Por lo tanto, esta función demanda el dominio de rasgos discursivos y gramaticales, pertenecientes a cada género y tipología textual.

Pero sin lugar a dudas, la función de la escritura que más nos compete en este estudio es la vinculada al ámbito académico, pues como sostiene Carlino (2006), escribir es uno de los métodos más poderosos para construir, crear y cambiar el mundo y la academia. Por eso, todas las asignaturas deben enfocarse en descubrir y desarrollar formas particulares de escribir y utilizar esta habilidad como estrategia compartida a la par de los contenidos de cada disciplina, conforme la tipología y género discursivo que circula en cada ámbito disciplinar.

\section{Escritura y procesos cognitivos}

Para la producción de un texto se requieren distintas actividades de pensamiento superior. Tales actividades conllevan una temporalidad que abarca desde el mismo momento en que se crea la circunstancia o situación social de escritura hasta la edición final de la producción. En ese sentido, y basándonos en la fundamentación teórico-práctica de Sanz (2010) sobre las competencias cognitivas, destacaremos el desarrollo de tres de los cinco niveles de las habilidades del pensamiento que deben implementarse para eficientizar destrezas comunicativas en los procesos de escritura. Estaremos enfocándonos en el pensamiento comprensivo, el pensamiento crítico y el pensamiento creativo.

El primer nivel de pensamiento (comprensivo), Villa y Poblete (2007) y Sanz (2010) lo han considerado como el modo de pensar que utilizamos para comprender la realidad, que a la vez estila un procedimiento operacional mediante niveles cognitivos destinados a favorecer la producción escrita mediante la comparación, la clasificación, el análisis, la síntesis, la secuenciación y el descubrimiento por razonamiento. Este nivel de pensamiento consiste en plantear situaciones en las que el estudiante utilice la comparación al servicio de la intertextualidad discursiva, como por ejemplo experimentar las semejanzas y diferencias que se establecen entre texto y contexto, así como entre el texto objeto de estudio y otros textos con los 
que de alguna manera y por alguna razón guardan relación (intertextualidad, polifonía discursiva). En lo que respecta a la clasificación, se procede a jerarquizar, a través de elementos clasificatorios, las informaciones que ofrece el texto, unas veces por el grado de importancia, o desde el aspecto estructural; otras veces por la intencionalidad del autor y, en ocasiones, desde la visión del lector. En relación al análisis y la síntesis, se establecen conjeturas, pareceres, posturas y puntos de vista como resultado del estudio y sus partes hasta llegar al todo, como resultado de la construcción de un nuevo concepto o pensamiento. Todo esto mediante un proceso racional que sustenta la posición argumentada desde la que se crea o construye una nueva producción.

Para el segundo nivel de pensamiento (crítico) se prevé que el estudiantado desarrolle una óptima capacidad de juicio y la utilice para solucionar múltiples problemas que tendrá que enfrentar. Además, este nivel de pensamiento se enfoca en descubrir la fiabilidad de la información que analiza (Sanz, 2010). En ese sentido, entendemos que comprender no es suficiente si no se tiene la capacidad de examinar el contenido con relevancia, profundidad, amplitud y sentido lógico. Al respecto, Campos (2007) postula que el pensamiento crítico es el pensar claro y racional que favorece el desarrollo del pensamiento reflexivo e independiente que permite a toda persona realizar juicios confiables sobre la credibilidad de una afirmación o la conveniencia de una determinada acción. Por su parte, Villa y Poblete (2007) establecen pautas o la rutina para fortalecer el pensamiento crítico: hacerse preguntas sobre la realidad; analizar la coherencia de los juicios propios y ajenos y argumentar la pertinencia de los juicios emitidos. Este nivel conjuga el desarrollo de cinco capacidades cognitivas vinculadas a las destrezas de pensamiento crítico: investigación, interpretación, predicción, razonamiento analógico y razonamiento deductivo (Sanz, 2010).

El desarrollo de la capacidad investigativa implica la aplicación de procesos de intertextualidad. Con ello se procura validar la fiabilidad de las fuentes de información, a partir de fuentes primarias diversas que coadyuven a valorar la procedencia de la información, objeto de análisis. Todo esto a partir de cuestionamientos, a modo de problema, que surgen en la mente del estudiante lectorescritor. Es así como se requiere la búsqueda de causas, y sus interpretaciones, a fin de descubrir y analizar el origen de lo acontecido, a partir de datos fiables y válidos, y así determinar qué causas serían las más probables. En lo adelante, no se hace esperar la predicción de los efectos, bien sea analizando probabilidades o proponiendo hipótesis inferenciales y verificables.

Para el tercer y último nivel de pensamiento (creativo) hemos intentado incorporar los niveles de pensamiento antes tratados (comprensivo y crítico), en virtud de la necesidad de generar información de forma creativa. En efecto, Boeglin (2008) advierte que para desarrollar esta línea de pensamiento es pertinente estar bien informado y, por supuesto, disponer de una actitud evaluativa ante cualquier evento de producción. Para ello, intentaremos describir, brevemente, las siguientes dimensiones cognitivas asociadas al pensamiento creativo: generación de ideas, establecimiento de relaciones, producción de imágenes, creación de metáforas y emprendimiento de metas (Sanz, 2010).

En lo que respecta a la generación de ideas, Escribano y Del Valle (2008) entienden que es conveniente construir creativamente respuestas, soluciones y sugerencias, en demanda a interrogantes formuladas por el estudiantado, unas veces al autor, otras veces al texto y otras a los diferentes contextos en que se recrea el discurso. Cuando se trata de crear producciones auténticas, entonces apelamos al establecimiento de relaciones con el propósito de descubrir y demostrar conexiones, comparando información procedente de diferentes fuentes y modalidades. Además, se construyen argumentos, analogías y se trabaja en la elocución del escrito. Para esto se han utilizado diversas técnicas y se han reformulado conceptos destinados a la mejora del nuevo texto que se ha producido.

Los niveles cognitivos planteados anteriormente nos revelan la interrelación existente entre lectura y escritura, dos acciones inseparables que requieren habilidades y destrezas cognitivas, más allá de las experiencias previas, las situaciones de lectura y escritura y el contenido o conocimiento que se pudiera tener del texto que se lee con miras a una nueva producción escrita. Por lo cual es 
necesario que los docentes nos dispongamos a desarrollar estas competencias, pues como opina Sanz (2010), existe la suposición tácita de que los estudiantes ya poseen estas destrezas de pensamiento, sin embargo, esta concepción es errónea, y resulta contraproducente que se les exija a los estudiantes analizar, sintetizar, emitir juicios, comparar o relacionar datos o posturas de distintos autores, sin antes desarrollar estas competencias en el aula a través de estrategias eficaces para conseguir tales fines.

\section{Modelos para enseñar y aprender a escribir}

Para referirnos a los distintos modelos de enseñanza y aprendizaje de la escritura, nos hemos basado en modelos desarrollados por distintos autores (Cassany, 2007; Milian y Camps, 2000; Graves, 2002; Avendaño y Desinano, 2007). Estos modelos nos ofrecen una propuesta dirigida a la enseñanza y al aprendizaje de la producción escrita. El aprendizaje de la escritura, así como sus implicaciones en las prácticas docentes más comunes, parten generalmente del aporte de tres modelos, que son: modelo del producto, modelo de procesos cognitivos y modelo de la escritura a través de las disciplinas.

En relación al modelo del producto, Graves (2002) advierte que los referentes teóricos de un modelo de producto los podemos encontrar en los textos de gramática tradicional, de retórica y estilística; en manuales de composición, entre otros materiales de esta naturaleza. Milian y Camps (2000) señalan que al texto escrito se le atribuye un carácter estático, refrendado por mecanismos codificadores de superficie, tales como la ortografía, la puntuación, la gramática y otros aspectos formales.

Este modelo, generalmente, es relacionado por algunos autores con métodos tradicionales, alusivos a criterios prescriptivos y gramaticales de la Real Academia de la Lengua Española. Cassany (2007), por ejemplo, lo reconoce como enfoque basado en la gramática. Avendaño y Desinano (2007) lo distinguen como una asimilación pasiva de los conocimientos, basada en la memorización y procedimientos rutinarios. Los estudios llevados a cabo por Marín (2008) muestran cómo este modelo ha impactado la producción escrita poniendo de manifiesto el énfasis en la gramática y normativa, que incluye el cuidado por las reglas de ortografía, morfosintaxis y léxico.

En lo que respecta al modelo de procesos cognitivos, las etapas y operaciones intelectuales necesarias que coadyuvan, de algún modo, a la producción textual escrita están estrechamente vinculadas a él. Según Lomas (2006), diversos estudiosos coinciden en los procesos de escritura, con varios puntos en común. Van Dijk (1978) orienta los procesos de reproducción, reconstrucción y elaboración textual. Flower y Hayes (1980) nos presentan las etapas: planificar, textualizar y revisar. Cassany (1989) reitera la coincidencia de las etapas, pero las denomina: pre-escribir, escribir y re-escribir. Contrario a los modelos orientados al producto, que prescriben una enseñanza del texto escrito a partir de normas gramaticales, en el modelo cognitivo se importantiza el escritor y sus procesos mentales: se atiende al escritor y luego al escrito.

En este modelo se observan prácticas que implican el desarrollo de operaciones mentales que abarcan desde la acción de recordar hasta crear. Estas prácticas Alvarez (2005) las clasifica en procesos cognitivos de orden inferior y procesos cognitivos de orden superior. En lo concerniente a los procesos cognitivos de orden inferior, se desarrollan procesos vinculados al recuerdo, a la comprensión y a la aplicación; mientras que los procesos de orden superior desarrollan operaciones mentales que abarcan desde el análisis hasta la creación. Cada una de estas operaciones son desarrolladas mediante preguntas cuyas acciones o resultados conducen a la implementación de la operación mental esperada, conforme a su naturaleza.

Cuando nos proponemos llegar a comprender, entonces procuramos mostrar entendimiento a la hora de encontrar información del texto. Es por esto que al acudir a preguntas que impliquen explicación, clasificación, comparación y contraste, entre otras interrogantes del pensamiento compresivo, estaríamos en condiciones de alcanzar esta operación mental. Y si nos proponemos examinar en detalle la información, identificando los motivos o causas, sin dudas estaríamos ante un proceso para el alcance del pensamiento crítico. En definitiva, estas operaciones mentales forman parte del modelo de procesos cognitivos y son las 
que han prevalecido en proyectos dirigidos a poner en práctica este modelo, tanto en los procesos de comprensión como en los de pensamiento crítico, cuyas operaciones mentales, además de las ya mencionadas, procuran justificar, ponderar y defender informaciones mediante juicios razonables hasta cambiar o crear proponiendo una nueva información y soluciones alternativas.

En cuanto al modelo de la escritura en las distintas disciplinas, los aportes de Carlino (2006) han colaborado para que la escritura en la universidad sea asumida en todas las asignaturas, y no solo sea vista como una práctica instrumental en las asignaturas remediales de la lengua que generalmente se ubican al inicio de las carreras. Por el contrario, propone un desarrollo de prácticas letradas con el fin de que el estudiante pase a formar parte de una comunidad discursiva que le permita familiarizarse con la nueva cultura escrita propia de los textos que circulan en la academia. El impacto de este modelo queda de manifiesto en los trabajos presentados por muchos investigadores, entre ellos, Carlino y Martínez (2009) y Natale (2013), quienes nos muestran las prácticas de lectura y escritura y su impacto en proyectos de escritura en la universidad.

La intención de presentar estos tres modelos no ha sido separarlos del desarrollo de procesos cognitivos. Por el contrario, como hemos visto, estos modelos implican la puesta en ejecución de destrezas cognitivas, pues si nos vamos al primero, aunque no es el más recomendado porque su mayor énfasis recae en prescripciones relativas a la normativa gramatical, es evidente que el recuerdo habrá de estar presente si queremos implementar el conocimiento lingüístico, además de que una de las propiedades del texto es la gramaticalidad. $Y$ en el caso del tercer modelo, a la hora de escribir, conforme a un género discursivo específico, hemos de tomar decisiones sobre qué, cómo y a quién escribir y resolver los problemas retóricos que conlleva la producción de un texto.

\section{Estrategias para guiar los procesos de la escritura}

Compartimos, a continuación, unas estrategias para la producción escrita que, desde nuestra experiencia y consideración, pueden guiar los procesos de enseñanza y aprendizaje de la escritura, con el fin de alcanzar producciones adecuadas y eficaces. Esta propuesta metodológica será útil para una didáctica de la composición. Con ella nos proponemos orientar las fases de escritura de un texto a la luz de los fundamentos contenidos en el presente estudio.

Un escrito es el producto de operaciones mentales e intelectuales. La enseñanza de la escritura requiere la valoración de lo que se escribe como el producto de un proceso largo y complejo, mediante una serie de operaciones que el estudiante ha de realizar. Dichas operaciones son agrupadas en las siguientes fases: pre-desarrollo, cuyo objetivo es estimular la creación, selección y organización de hechos e ideas; desarrollo verdadero, que implica un delicado trabajo de transformación de las ideas en un texto; reunión de reacciones y comentarios sobre el desarrollo, no solo por parte del docente, además puede ser por parte de compañeros y familiares; revisión, que incluye reescrituras y comentarios y, finalmente, la composición, que radica en la presentación o versión final del escrito. (Serafini, 1997).

A partir de los planteamientos y experiencias ofrecemos una propuesta que apunta al desarrollo de procesos cognitivos que orientan el desarrollo de la producción escrita en los tres niveles de pensamiento en que nos hemos enfocado: pensamiento comprensivo, pensamiento crítico y pensamiento creativo (Sanz, 2010). Cada uno de estos niveles los presentamos vinculados a las distintas etapas del modelo de Flower y Hayes (1980). Con esta propuesta esperamos que el estudiantado logre desarrollar capacidad para procesar e interpretar información de manera reflexiva y precisa y pueda lograr habilidades que le permita resolver situaciones y mediar conflictos a través del uso razonado y argumentado de la palabra y de esa manera estar en condiciones para analizar y examinar en detalle la información que habrá de ser emitida con claridad, objetividad y pertinencia. También apostamos al desarrollo de capacidades para realizar inferencias y llegar a conclusiones precisas tras un conjunto de informaciones diversas y de este modo, crear, construir y aportar nuevas informaciones que se conviertan en novedosos conocimientos. 
Tabla 1: Competencias cognitivas para desarrollar el pensamiento comprensivo al producir un ensayo. Etapa de planificación

\begin{tabular}{|c|c|c|c|}
\hline Destrezas de pensamiento & Estrategias & Resultados de aprendizaje & Actividades \\
\hline $\begin{array}{l}\text { Comparar, clasificar, analizar, } \\
\text { sintetizar, secuenciar, } \\
\text { descubrir }\end{array}$ & $\begin{array}{l}\text { Aplicar los procesos inferenciales } \\
\text { pertinentes para una adecuada } \\
\text { comprensión escrita de un texto } \\
\text { mediante el empleo de reglas } \\
\text { que capturan el sentido global } \\
\text { del texto. } \\
\text { Producir resúmenes y mapas } \\
\text { conceptuales como insumos } \\
\text { para su futuro texto. }\end{array}$ & $\begin{array}{l}\text { Establece comparación entre dos } \\
\text { realidades, a partir de sus semejanzas y } \\
\text { diferencias. } \\
\text { Clasifica diversos elementos, } \\
\text { agrupándolos conforme a categorías } \\
\text { específicas. } \\
\text { Sintetiza el contenido de un texto a partir } \\
\text { de su secuencia y estructura } \\
\text { Determina el mensaje o idea global que } \\
\text { quiso expresar el autor de un determinado } \\
\text { texto. }\end{array}$ & $\begin{array}{l}\text { Búsqueda de información } \\
\text { Análisis bibliográfico } \\
\text { Lecturas guiadas } \\
\text { Grupos de discusión }\end{array}$ \\
\hline
\end{tabular}

(Fuente: Elaboración propia a partir de los planteamientos de Sanz, 2010)

Tabla 2: Pasos para desarrollar el pensamiento crítico al producir un ensayo. Etapa de textualización

\begin{tabular}{|c|c|c|c|}
\hline $\begin{array}{l}\text { Destrezas de } \\
\text { pensamiento }\end{array}$ & Estrategias & Resultados de aprendizaje & Actividades \\
\hline $\begin{array}{l}\text { Investigar la fiabilidad de } \\
\text { las fuentes } \\
\text { Interpretar causas } \\
\text { Predecir efectos } \\
\text { Razonar analógicamente } \\
\text { Razonar deductivamente }\end{array}$ & $\begin{array}{l}\text { Producir textos a partir de } \\
\text { diversas fuentes, tomando en } \\
\text { cuenta sus rasgos lingüístico- } \\
\text { discursivos, la situación en la } \\
\text { que se enuncian y los procesos } \\
\text { estratégicos que involucra su } \\
\text { realización. }\end{array}$ & $\begin{array}{l}\text { Investiga la fiabilidad de la fuente de } \\
\text { información que se está evaluando. } \\
\text { Distingue fuentes primarias y } \\
\text { secundarias. } \\
\text { Interpreta las causas y los efectos } \\
\text { inherentes a un hecho o situación } \\
\text { determinada. } \\
\text { Interpreta analógicamente procedimientos } \\
\text { que coadyuvan a la solución de } \\
\text { problemas o situaciones concretas. } \\
\text { Deduce, mediante la estructura de un } \\
\text { argumento, la conclusión a la que puede } \\
\text { Ilegarse, tras leer un determinado texto. } \\
\text { Produce un texto coherente sobre la } \\
\text { temática elegida e incluye diferentes } \\
\text { fuentes para la fundamentación de las } \\
\text { ideas. }\end{array}$ & $\begin{array}{l}\text { Búsqueda de información } \\
\text { Análisis intertextual } \\
\text { Lecturas guiadas } \\
\text { Grupos de discusión } \\
\text { Elaboración de borradores a } \\
\text { partir de una consigna con } \\
\text { especificaciones claras sobre el } \\
\text { texto que está produciendo. }\end{array}$ \\
\hline
\end{tabular}

(Fuente: Elaboración propia a partir de los planteamientos de Sanz, 2010) 
Tabla 3: Pasos para desarrollar el pensamiento creativo al producir un ensayo. Etapa de revisión

\begin{tabular}{|c|c|c|c|}
\hline $\begin{array}{l}\text { Destrezas de } \\
\text { pensamiento }\end{array}$ & Estrategias & Resultados de aprendizaje & Actividades \\
\hline $\begin{array}{l}\text { Generar ideas } \\
\text { Establecer relaciones } \\
\text { Producir imágenes } \\
\text { Crear metáforas o } \\
\text { analogías } \\
\text { Emprender metas }\end{array}$ & $\begin{array}{l}\text { Producir textos académicos } \\
\text { que respondan a un género } \\
\text { específico tomando en } \\
\text { cuenta sus rasgos lingüístico- } \\
\text { discursivos, la situación en la } \\
\text { que se enuncian y los procesos } \\
\text { estratégicos que involucra su } \\
\text { realización. } \\
\text { Revisar y editar textos. } \\
\text { Trabajar el estilo para que } \\
\text { el producto escrito logre } \\
\text { los objetivos comunicativos } \\
\text { deseados. }\end{array}$ & $\begin{array}{l}\text { Propone ideas, sugerencias o soluciones } \\
\text { ante una situación o problemática } \\
\text { determinada. } \\
\text { Establece relaciones y comparaciones } \\
\text { entre dos o más realidades afines u } \\
\text { opuestas. } \\
\text { Produce frases o expresiones metafóricas } \\
\text { o analógicas vinculadas a hechos o } \\
\text { situaciones de la cotidianidad en las que } \\
\text { se traslade el sentido real de un objeto, } \\
\text { concepto o suceso. } \\
\text { Evalúa objetivamente otros textos } \\
\text { para identificar su estructura global y } \\
\text { estrategias discursivas. }\end{array}$ & $\begin{array}{l}\text { Trabajo colaborativo para la } \\
\text { revisión del código escrito y del } \\
\text { género discursivo a través de } \\
\text { rúbricas y } \\
\text { listas de cotejo. } \\
\text { Revisión individual para la } \\
\text { mejora en cuanto a la resolución } \\
\text { de problemas retóricos. }\end{array}$ \\
\hline
\end{tabular}

(Fuente: Elaboración propia a partir de los planteamientos de Sanz, 2010)

\section{Conclusión}

Sin dudas, los procesos de producciones escritas resultan sumamente complejos, pues van más allá de la aplicación de saberes o habilidades instrumentales y prescriptivas que en ocasiones no pasan de ser transcripciones informativas, rutinarias y mecánicas carentes de sentido y ajenas a todo proceso cognitivo. Si bien la producción escrita, al igual que otros actos comunicativos, no siempre se enmarca en un mismo contexto, circunstancia o situación, siempre habrá de por medio unas acciones necesarias para establecer el proceso que conlleva este acto de carácter social y cultural, creado por la humanidad con fines eminentemente comunicativos y en los que intervienen variadas estrategias y la motivación para lograrlo.

Al inicio de este artículo nos proponíamos exponer el impacto de los procesos cognitivos necesarios para los productos escritos a través del uso de estrategias que potencian las destrezas de pensamiento, pues el acto de escribir no escapa de habilidades mentales que incluyen el recuerdo y los referentes que conectan directamente con otros mundos y escenarios que nos proveen nociones e información, objeto de comprensión y análisis, con fines prácticos para la producción de un texto. Parecería que con esto es suficiente; sin embargo, será necesario asumir un grado de conciencia, producto del conocimiento y la experiencia, que nos permita valorar el producto, mediante un proceso de análisis, y solo así estaremos en condiciones de plasmar nuestras ideas en un texto de carácter original y creativo, conforme a necesidades e intereses comunicativos, sin olvidar las expectativas del destinatario a quien dirigimos el mensaje.

Se espera que esta propuesta impacte las estrategias y actividades de aprendizaje de la escritura, permitiendo que el docente provoque espacios críticos, analíticos y reflexivos en el aula, de modo que la asignatura sea un espacio de acción y construcción de conocimientos por medio de la producción escrita. Conocidas las complejidades de la escritura, es necesario que los estudiantes reciban las orientaciones pertinentes a través de consignas claras; se fortalezca el proceso de redacción y que en cada una de las etapas se incluyan las competencias cognitivas necesarias que desarrollen la capacidad de producir textos 
adecuados a circunstancias y contextos reales y ello posibilite a los estudiantes su inserción en las comunidades discursivas propias de sus disciplinas. En definitiva, apostamos a un estudiantado crítico, analítico, reflexivo y creador que no solo se limite a recibir y repetir información, sino a crear nuevas expresiones con libertad, creatividad y originalidad.

\section{Referencias}

Alvarez, J. (2005). Teoría lingüística y enseñanza de la lengua. Madrid, España: Greolf.

Avendaño, F. y Desinano, N. (2007). Didácticas de las Ciencias del Lenguaje. Buenos Aires, Argentina: Homo Sapiens.

Boeglin, M. (2008). Leer y redactar en la universidad. Bogotá, Colombia: Editorial Magisterio.

Calsamiglia, H. y Tusón, A. (2009). Las cosas del decir. Barcelona, España: Ariel Lingüística.

Camps, A. (2000). El papel de la actividad metalingüística en el aprendizaje de la escritura. Santa Fe, Argentina: HomoSapiens.

Camps, A. (2003). Secuencias didácticas para aprender a escribir. Barcelona, España: Graó.

Campos, A. (2007). Pensamiento crítico: Técnicas para su desarrollo. Bogotá, Colombia: Editorial Magisterio.

Carlino, P. (2006). Escribir, leer y aprender en la universidad. Buenos Aires, Argentina: Fondo de Cultura Económica.

Carlino, P. y Martínez, S. (2009). La lectura y la escritura: un asunto de todos. Buenos Aires, Argentina: Universidad Nacional del Comahue.

Cassany, D. (2007). Enseñar lengua. Barcelona, España: Graó.

Cassany, D. (1989). Describir el escribir. Barcelona, España: Paidós.

Cisneros, M. (2014). Perspectiva y prospectiva en los estudios sobre lectura y escritura. Colombia: Universidad Tecnológica de Pereira.
Coulmas, F. (2006). The Blackwell Encyclopedia of Writing Systems Inicia. Oxford, Cambridge: University Press

Escribano, A. y Del Valle, A. (2008). El aprendizaje basado en problemas. Madrid, España: Narcea.

Flower, L. y Hayes, J.R. (1980). A Cognitive Process Theory of Writing. College Composition and Communication, 32, 365387. http://dx.doi.org/10.2307/356600

Graves, D. (2002). Didáctica de la escritura. Madrid, España: Centro de Publicaciones, Ciudad Universitaria.

Lomas, C. (2006). Enseñar lenguaje para aprender a comunicarse. Bogotá, Colombia: Magisterio, Editorial.

Marinkovich, J. (2002). Enfoques de proceso en la producción de textos escritos. Valparaíso: Revista signos. V.35 n.51-52.

Milian, M. y Camps, A. (2000). El papel de la actividad metalingüística en el aprendizaje de la escritura. Rosario (Santa Fe), Argentina: HomoSapiens.

Marín, M. (2008). Lingüística y enseñanza de la lengua. Buenos Aires, Argentina: Aique.

Natale, L. (Coord.) (2012). El semillero de la escritura. Buenos Aires, Argentina: Universidad Nacional de General Sarmiento.

Sánchez, L. (2006). Saber escribir. Madrid, España: Instituto Cervantes.

Sanz, M. (2010). Competencias cognitivas en educación superior. Madrid, España: Narcea.

Serafini, M. (1997). Cómo redactar un tema. Barcelona: Paidós.

Van Dijk, T. (1978). La ciencia del texto. Barcelona: Paidós.

Villa, A. y Poblete, M. (2007). Aprendizaje basado en competencias. Universidad de Deusto: Bilbao. 